\title{
Pharmacokinetics and Dialyzability of a Single Oral Dose of Amenamevir, an Anti-Herpes Drug, in Hemodialysis Patients
}

\author{
Shuichi Tsuruoka · Takamasa Endo (D) - Mizuna Seo · Naoto Hashimoto
}

Received: April 1, 2020 / Published online: May 21, 2020

(C) The Author(s) 2020

\begin{abstract}
Introduction: Amenamevir (ASP2151), a herpesvirus helicase-primase inhibitor, is currently used for the treatment of herpes zoster in Japan. Amenamevir is mainly metabolized in the liver, and urinary excretion of amenamevir is approximately $10 \%$ in healthy adults. The increase of systemic exposure in non-dialysis patients with severe renal impairment was much less than that associated with nucleoside antiviral agents. The aim of this study was to evaluate the pharmacokinetics and dialyzability of a single oral dose ( $400 \mathrm{mg}$ ) of amenamevir in hemodialysis patients.
\end{abstract}

Digital Features To view digital features for this article go to https://doi.org/10.6084/m9.figshare.12229205.

Electronic Supplementary Material The online version of this article (https://doi.org/10.1007/s12325020-01375-1) contains supplementary material, which is available to authorized users.

S. Tsuruoka

Department of Nephrology, Nippon Medical School,

Tokyo, Japan

T. Endo $(\bowtie) \cdot$ M. Seo

Clinical Development Department, Maruho Co.,

Ltd., Kyoto, Japan

e-mail: endou_cmn@mii.maruho.co.jp

N. Hashimoto

Drug Development Research Laboratory, Maruho

Co., Ltd., Kyoto, Japan
Methods: This was a single-arm, open-label, multicenter clinical pharmacology study. Nine patients aged 20-80 years with end-stage kidney disease and undergoing maintenance hemodialysis three times weekly were enrolled. Pharmacokinetics and dialyzability were investigated by serial collection of blood samples until $48 \mathrm{~h}$ post-dose during the study.

Results: The maximum plasma concentration and time to reach maximum plasma concentration during $24 \mathrm{~h}$ post-dose were $1585 \mathrm{ng} / \mathrm{mL}$ and $6.2 \mathrm{~h}$, respectively. The area under the plasma concentration-time curve (AUC) from time zero to $24 \mathrm{~h}$ was $23,890 \mathrm{ng} \mathrm{h} / \mathrm{mL}$. The median terminal elimination half-life within $24 \mathrm{~h}$ before, during, and after hemodialysis was $14.7,15.2$, and $12.4 \mathrm{~h}$, respectively. The AUC in hemodialysis patients was approximately double that in healthy adults. This increase in AUC was much less than that reported in nucleoside antiviral agents. The hemodialysis clearance, elimination fraction percentage, and amount of amenamevir removed were $37.8 \mathrm{~mL} / \mathrm{min}$, $28.1 \%$, and $132.0 \mu \mathrm{g}$, respectively. The amount of amenamevir removed by hemodialysis was minimal. None of the hemodialysis parameters were associated with serum albumin. This study revealed no clinically relevant safety concerns.

Conclusion: There were no clinically relevant safety concerns when $400 \mathrm{mg}$ of amenamevir was administered as a single dose to hemodialysis patients without dose adjustment and/or modification of the dosing schedule. 
Trial Registration: JapicCTI-184242.

Keywords: Amenamevir; Helicase-primase inhibitor; Hemodialysis; Herpes zoster; Nephrology; Pharmacokinetics

\section{Key Summary Points}

Why carry out this study?

Amenamevir (ASP2151), a herpesvirus helicase-primase inhibitor currently used for the treatment of herpes zoster in Japan, is mainly metabolized in the liver and urinary excretion of amenamevir was approximately $10 \%$ of the total dose in healthy adults.

The increase of systemic exposure in nondialysis patients with severe renal impairment was much less than that associated with nucleoside antiviral agents.

The aim of this study was to evaluate the pharmacokinetics and dialyzability of a single oral dose $(400 \mathrm{mg})$ of amenamevir in hemodialysis patients.

\section{What was learned from the study?}

The area under the plasma concentration-time curve (AUC) in hemodialysis patients was approximately double that in healthy adults, but the increase was much less than that reported in nucleoside antiviral agents.

The amount of amenamevir removed by hemodialysis was minimal.

There were no clinically relevant safety concerns when $400 \mathrm{mg}$ of amenamevir was administered as a single dose to hemodialysis patients without dose adjustment and/or modification of the dosing schedule.

\section{INTRODUCTION}

Amenamevir (ASP2151) is a non-nucleoside human herpesvirus helicase-primase inhibitor [1] that was approved at a dosage of $400 \mathrm{mg}$ once a day for the treatment for herpes zoster (shingles) in Japan in 2017. Phase 1 studies of amenamevir in healthy adults demonstrated that amenamevir is safe and well tolerated from 5 to $2400 \mathrm{mg}$ as a single dose and from 300 to $600 \mathrm{mg}$ as 7-day repeated doses [2]. Amenamevir is mainly metabolized in the liver $[3,4]$ and urinary excretion of unchanged amenamevir was approximately $10 \%$ in healthy adults [5]. In patients with chronic kidney disease (CKD), the clearance of amenamevir decreased depending on the severity of renal failure [6]. However, even in non-dialysis patients with severe renal impairment, systemic exposure was found to be 1.78 times higher than that of healthy adults, and amenamevir was safe and well tolerated in these patients [6]. Therefore, the package insert of amenamevir does not require dose reduction in relation to the degree of renal impairment [7]. On the other hand, nucleoside antiviral agents such as acyclovir [8], valaciclovir [9], and famciclovir [10] are mainly excreted by the kidney; therefore, dose adjustment is needed on the basis of the creatinine clearance (Ccr) in patients with CKD. In general, there would be higher risk of drug accumulation in patients with end-stage kidney disease (ESKD) that require renal replacement therapy such as hemodialysis than in non-dialysis patients with CKD. In the former patients, dose reduction is often needed to avoid adverse drug reactions [11]. In addition, drug dialyzability and its potential impact on drug efficacy should be considered in hemodialysis patients. If removal of a drug by dialysis is significant, additional dosage or modification of the dosing schedule would be required to ensure adequate therapeutic efficacy [12]. However, there is no report describing the pharmacokinetics and dialyzability of amenamevir in hemodialysis patients.

Here, we report the pharmacokinetics and dialyzability of amenamevir in hemodialysis patients, when $400 \mathrm{mg}$ was orally administered, 
which is used for the treatment of herpes zoster in patients with normal renal function.

\section{METHODS}

\section{Study Design}

This was a single-arm, open-label, multicenter clinical pharmacology study in patients undergoing maintenance hemodialysis to evaluate the pharmacokinetics and dialyzability of amenamevir and its major metabolite R5 when a single dose $(400 \mathrm{mg})$ of amenamevir was administered orally after breakfast. The study protocol and informed consent form were approved by the institutional review board at each participating study site (Supplementary Information). All patients gave written informed consent before initiation of any study specific procedures. The study was designed and conducted by the sponsor (Maruho Co., Ltd.) in collaboration with the principal investigators. The sponsor monitored study conduct, collected the data, and performed the statistical analyses. The study was conducted in accordance with the ethical principles originating in or derived from the Declaration of Helsinki, International Conference on Harmonisation Good Clinical Practice Guidelines, and locally applicable laws and regulations. This study is registered with the Japan Pharmaceutical Information Center; JapicCTI-184242.

\section{Patients and Treatment}

Male and female patients (eight males and one female) aged 20-80 years with a diagnosis of ESKD undergoing 4-h maintenance hemodialysis using a polysulfone dialyzer membrane three times weekly for at least 12 weeks were enrolled in the study. Other inclusion criteria were dry weight between 40.0 and $80.0 \mathrm{~kg}$, and body mass index between 16.0 and $30.0 \mathrm{~kg} / \mathrm{m}^{2}$. Key exclusion criteria were patients who had significant cardiac, hepatic, pulmonary, or blood disease that made them unsuitable for participation in clinical trials; hepatitis B virus, hepatitis $\mathrm{C}$ virus, or HIV infection; hemoglobin level less than $9 \mathrm{~g} / \mathrm{dL}$ at screening; and history of gastrointestinal surgery.

Patients received a 400-mg single oral dose of amenamevir (two 200-mg tablets) $30 \mathrm{~min}$ after breakfast on day 1 in the morning (Fig. 1). During the study, patients were hospitalized at the study site from day -1 to day 3 (three nights/four days), and received 4-h hemodialysis on day -1 and $24 \mathrm{~h}$ post-dose on day 2 . Use of new drugs, or a change in the dose or regimen of the concomitant drugs used before day -14 , or change in surgical or physical therapies was prohibited during the period from day -14 to day 3. Prohibited concomitant medications and therapies comprised CYP3A inhibitors, which may increase amenamevir blood concentration, including macrolide antibiotics, azole antifungal agents, HIV protease inhibitors, other drugs such as danazol, tofisopam, bromocriptine mesylate, fluvoxamine maleate, delavirdine mesylate, etc.; citrus containing a large amount of furanocoumarins such as grapefruit, sweetie, pomelo, etc.; CYP3A inducers, which may decrease amenamevir blood concentration, including rifampicin, carbamazepine, barbiturates, hydantoin antiepileptics, amobarbital, nevirapine; and food containing St. John's wort. Drugs that are metabolized by CYP3A could be used in conjunction with amenamevir, but were to be administered carefully while remaining alert to the possibility of adverse events during the period of concomitant administration.

\section{Study Assessments}

Blood samples for the measurement of amenamevir and R5 concentration in plasma were taken immediately pre-dose and then $0.25,0.5$, $1,1.5,2,3,4,6,8,12,24,25,26,28,31,34$, and $48 \mathrm{~h}$ post-dose. Blood samples for the measurement of protein binding of amenamevir were collected 4 and $24 \mathrm{~h}$ post-dose. Blood samples for dialyzability assessment were collected from healthy veins other than the arteriovenous shunt. On day 2 , during hemodialysis treatment that started $24 \mathrm{~h}$ post-dose, blood samples were taken from the inlet and outlet of the dialyzer at $24,25,26$, and $28 \mathrm{~h}$ post-dose. The hematocrit 


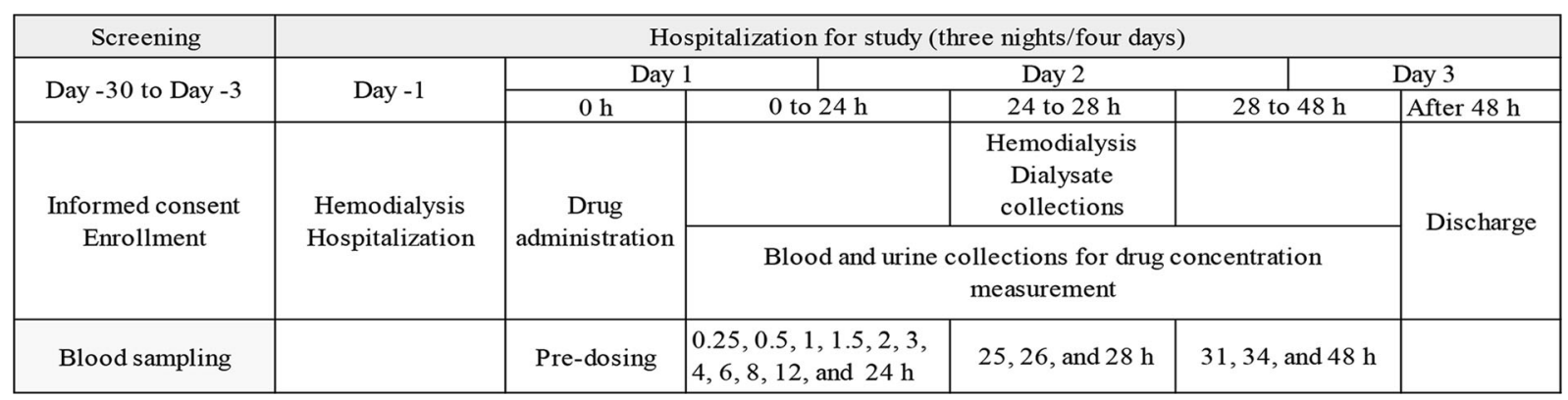

Fig. 1 Study design and schedule

used for the permeability estimation was also measured. Twelve-hour urine samples were collected $0-24$ and $24-48 \mathrm{~h}$ post-dose. Plasma, urine, and dialysate concentration of amenamevir and R5 were measured by validated LC/ MS/MS. The lower limits of quantitation (LLOQ) of amenamevir in plasma, urine, and dialysate were $5.0,50$ and $2.5 \mathrm{ng} / \mathrm{mL}$, respectively. Those of R5 in plasma, urine, and dialysate were $2.0,20$, and $1.0 \mathrm{ng} / \mathrm{mL}$, respectively. Pharmacokinetic assessments consisted of amenamevir and R5 concentrations in plasma, plasma at the inlet and outlet of the dialyzer, urine, and dialysate. The pharmacokinetic parameters were calculated on the basis of amenamevir and R5 concentrations in plasma by a non-parametric superposition method using Phoenix WinNonlin Standard version 6.3 (Pharsight Corporation, Sunnyvale, CA, USA). Pharmacokinetic parameters for amenamevir and R5 included maximum plasma concentration $\left(C_{\max }\right)$, time to reach maximum plasma concentration during $24 \mathrm{~h}$ post-dose $\left(t_{\max }\right)$, AUC from time zero to $24 \mathrm{~h}\left(\mathrm{AUC}_{24 \mathrm{~h}}\right), \mathrm{AUC}$ from time zero to infinity $\left(\mathrm{AUC}_{\mathrm{inf}}\right)$, terminal elimination half-life $\left(t_{1 / 2}\right)$ observed within $24 \mathrm{~h}$ $\left(t_{1 / 2 \mathrm{pre}}\right), t_{1 / 2}$ during hemodialysis $\left(t_{1 / 2 \mathrm{HD}}\right), t_{1 / 2}$ after hemodialysis (t $\left.t_{1 / 2 \text { post }}\right)$, AUC during hemodialysis $\left(\mathrm{AUC}_{\mathrm{D}}\right), \mathrm{AUC}$ during hemodialysis calculated on the basis of the concentration at the inlet of the dialyzer $\left(\mathrm{AUC}_{\mathrm{A} 24-28 \mathrm{~h}}\right)$, and AUC during hemodialysis calculated on the basis of the concentration at the outlet of the dialyzer $\left(\mathrm{AUC}_{\mathrm{V} 24-28 \mathrm{~h}}\right)$. Urine pharmacokinetic parameters from time zero to $24 \mathrm{~h}\left(\mathrm{Ae}_{24 \mathrm{~h}}\right)$ and from time 24 to $48 \mathrm{~h}\left(\mathrm{Ae}_{24-48 \mathrm{~h}}\right)$ were calculated for both amenamevir and R5. The hemodialysis parameters assessed were hemodialysis clearance $\left(\mathrm{CL}_{\mathrm{HD}}\right) \quad(\mathrm{mL} / \mathrm{min})$ [13], hemodialysis clearance normalized with dialyzer surface area $\left(\mathrm{mL} / \mathrm{min} / \mathrm{m}^{2}\right)$ [14], elimination fraction percentage (EP) (\%) [14], and amount removed of amenamevir $(\mu \mathrm{g})$ [14]. The correlation between hemodialysis parameters and serum albumin or normalized dialysis dose $(K t / V)$ was assessed. The Ccr was estimated using the Cockcroft-Gault method and $K t / V$ was calculated using the Daugirdas method [15].

The formulae used to calculate $\mathrm{CL}_{\mathrm{HD}}(\mathrm{mL} /$ min) (Eq. 1), hemodialysis clearance normalized with dialyzer surface area (Eq. 2), EP (\%) (Eq. 3), and amount removed of amenamevir $(\mu \mathrm{g})$ (Eq. 4) are shown below:

$$
\begin{aligned}
& \mathrm{CL}_{\mathrm{HD}}(\mathrm{mL} / \mathrm{min})=Q_{\mathrm{b}} \times\left(1-H_{\mathrm{A}}\right) \\
& \quad \times\left[C_{\mathrm{A}}-C_{\mathrm{V}} \times\left\{1-\left(H_{\mathrm{V}}-H_{\mathrm{A}}\right) / H_{\mathrm{V}}\right\}\right] / C_{\mathrm{A}},
\end{aligned}
$$

Hemodialysis clearance normalized with

$$
\begin{aligned}
& \text { dialyzer surface area }\left(\mathrm{mL} / \mathrm{min} / \mathrm{m}^{2}\right) \\
& \begin{array}{c}
Q_{\mathrm{b}} \times\left(1-H_{\mathrm{A}}\right)\left[C_{\mathrm{A}}-C_{\mathrm{V}}\right. \\
\left.\times\left\{1-\left(H_{\mathrm{V}}-H_{\mathrm{A}}\right) / H_{\mathrm{V}}\right\}\right] / C_{\mathrm{A}} / S \\
\mathrm{EP}(\%)=100 \times\left[1-C_{\mathrm{V}}\right. \\
\left.\times\left\{1-\left(H_{\mathrm{V}}-H_{\mathrm{A}}\right) / H_{\mathrm{V}}\right\} / C_{\mathrm{A}}\right]
\end{array}
\end{aligned}
$$

Amount of amenamevir removed $(\mu \mathrm{g})$

$$
=\left(\mathrm{AUC}_{\mathrm{A} 24-28 \mathrm{~h}}-\mathrm{AUC}_{\mathrm{V} 24-28 \mathrm{~h}}\right) \times Q_{\mathrm{b}}
$$

AUC $_{\text {A24-28h }}$ is the AUC calculated by trapezoidal method based on $C_{\mathrm{A}}$ during dialysis, 
AUC $_{\mathrm{V} 24-28 \mathrm{~h}}$ is the AUC calculated by trapezoidal method based on $C_{\mathrm{V}}$ during dialysis, $Q_{\mathrm{b}}$ is the dialyzer blood flow, $H_{\mathrm{A}}$ is the hematocrit value at dialyzer inlet, $H_{\mathrm{V}}$ is the hematocrit value at dialyzer outlet, $C_{\mathrm{A}}$ is the amenamevir or R5 concentration in plasma at dialyzer inlet, $C_{\mathrm{V}}$ is the amenamevir or R5 concentration in plasma at dialyzer outlet, $S$ is the dialyzer surface area.

The safety of amenamevir was assessed on the basis of adverse events (AEs), clinical laboratory evaluations (hematology and chemistry), vital sign measurements (axillary temperature, pulse, and supine systolic and diastolic blood pressure), and physical examinations. The investigators asked patients how they felt. The patients were also asked to self-report adverse events at any point in time to any staff member including the investigator and research nurse.

\section{Statistical Analyses}

Approximately six to ten patients are needed to evaluate the pharmacokinetics. Accordingly, ten patients were considered sufficient for achieving the study objectives. All safety analyses were conducted in all patients who received amenamevir. All pharmacokinetic analyses were performed in patients for whom sufficient plasma concentration data were available to facilitate the derivation of at least one pharmacokinetic parameter.

To assess the correlation between the hemodialysis parameters and serum albumin or $K t / V$, we used the Pearson's correlation coefficient $(r)$. Scatter diagrams were graphed to determine whether a linear trend existed.

AEs were coded using the Medical Dictionary for Regulatory Activities Japanese version (MedDRA/J), version 22.0.

\section{RESULTS}

\section{Patient Characteristics}

The study was conducted at six sites in Japan from February 2019 to June 2019. Among the ten patients who gave informed consent, nine patients met the eligibility criteria, and received a single oral 400-mg dose of amenamevir within 30 min following breakfast in the morning on day 1 (Fig. 1). Patient demographics and clinical characteristics at baseline are shown in Table 1. Eight male patients and one female patient with a mean age of 65.0 [standard deviation (SD) 9.0] years were enrolled in this study. The mean estimated Ccr (SD) and normalized dialysis dose (SD) $(K t / V)$ were $5.9(1.6) \mathrm{mL} / \mathrm{min}$ and 1.41 $(0.20)$, respectively. The hemodialysis prescriptions on day 2 for each patient are shown in Table 2.

\section{Pharmacokinetics}

The mean plasma concentration-time profiles of amenamevir and R5 are shown in Fig. 2. Summary statistics for the amenamevir and R5 pharmacokinetic parameters are shown in Table 3. The mean (SD) $\mathrm{AUC}_{24 \mathrm{~h}}$ and $\mathrm{AUC}_{\text {inf }}$ of amenamevir were 23,890 (8400) and 38,120 $(17,180) \mathrm{ng} \mathrm{h} / \mathrm{mL}$, respectively. The mean $\mathrm{AUC}_{24 \mathrm{~h}}$ of R5 was 6054 (2125) $\mathrm{ng} \mathrm{h} / \mathrm{mL}$. The median (range) $t_{1 / 2 \text { pre, }} t_{1 / 2 \mathrm{HD}}$, and $t_{1 / 2 \text { post }}$ of amenamevir were $14.7 \quad(9.9-17.0), 15.2$ (7.5-158.3), and 12.4 (8.4-15.7) h, respectively. The median (range) $t_{1 / 2 \mathrm{HD}}$ and $t_{1 / 2 \text { post }}$ of R5 were 11.9 (7.0-49.8) and $15.0(9.3-17.2) \mathrm{h}$, respectively. The mean (SD) $\mathrm{AUC}_{\mathrm{D}}, \mathrm{AUC}_{\mathrm{A} 24-28 \mathrm{~h}}$, and AUC $_{\mathrm{V} 24-28 \mathrm{~h}}$ of amenamevir were 2301 (926) $\mathrm{ng} \mathrm{h} / \mathrm{mL}, 2145$ (905) ng h/mL, and 1509 (672) $\mathrm{ng} \mathrm{h} / \mathrm{mL}$, respectively. The difference between $\mathrm{AUC}_{\mathrm{A} 24-28 \mathrm{~h}}$ and $\mathrm{AUC}_{\mathrm{V} 24-28 \mathrm{~h}}$ suggests that about $30 \%$ of amenamevir was removed by hemodialysis. The plasma concentration-time curve of multiple doses of amenamevir was estimated on the basis of the available data of seven patients under the assumption that no drug was removed by hemodialysis (Fig. 3). The mean cumulative amount of amenamevir and R5 excreted (Ae) in the urine from time zero to $48 \mathrm{~h}\left(\mathrm{Ae}_{48 \mathrm{~h}}\right)$ was 2.0 (1.1) $\mathrm{mg}$ and 1.4 (1.0) mg, respectively.

The dialyzability of amenamevir and its major metabolite R5 is shown in Table 4. The correlations between the dialyzability of amenamevir and either $K t / V$ or serum albumin are shown in Supplemental Fig. S1A-F. There was no correlation between $\mathrm{CL}_{\mathrm{HD}}$ and $K t / V(\mathrm{~A})$, but a 
Table 1 Individual patient demographics and clinical characteristics at baseline

\begin{tabular}{lllllll}
\hline Subject & Sex & Age (years) & Dry weight $(\mathbf{k g})$ & $\begin{array}{l}\text { Duration of dialysis } \\
(\mathbf{m o n t h s})\end{array}$ & $\begin{array}{l}\text { Creatinine clearance } \\
(\mathbf{m L} / \mathbf{m i n})\end{array}$ & $\begin{array}{l}\text { Albumin } \\
(\mathbf{g} / \mathbf{d L})\end{array}$ \\
\hline 1 & Male & 69 & 56.3 & 13.3 & 5.3 & 3.40 \\
2 & Female & 57 & 76.4 & 41.9 & 9.2 & 3.90 \\
3 & Male & 61 & 71.9 & 61.3 & 7.1 & 3.90 \\
4 & Male & 73 & 47.4 & 147.6 & 4.6 & 3.60 \\
5 & Male & 77 & 64.5 & 141.6 & 6.1 & 3.20 \\
6 & Male & 50 & 79.7 & 140.5 & 6.0 & 3.87 \\
7 & Male & 63 & 50.7 & 93.1 & 3.7 & 3.80 \\
8 & Male & 72 & 58.2 & 10.2 & 5.9 & 3.70 \\
9 & Male & 65 & 61.6 & 45.1 & 5.6 & 3.70 \\
Mean (SD) & - & $65.0(9.0)$ & $63.0(11.2)$ & $77.2(55.3)$ & $5.9(1.6)$ & $3.7(0.2)$ \\
\hline
\end{tabular}

$S D$ standard deviation

Table 2 Hemodialysis prescription of the individual patients on day 2

\begin{tabular}{llllllll}
\hline Subject & $\begin{array}{l}\text { Time from dosing } \\
\text { to start of HD }(\mathbf{h})\end{array}$ & $\begin{array}{l}\text { Duration of } \\
\text { HD session } \\
(\mathbf{h})\end{array}$ & $\begin{array}{l}\text { Blood flow } \\
\text { rate }(\mathbf{m L} / \\
\mathbf{m i n})\end{array}$ & $\begin{array}{l}\text { Ultrafiltration } \\
\text { rate }(\mathbf{L} / \mathbf{h})\end{array}$ & $\begin{array}{l}\text { Dialyzer } \\
\text { membrane }\end{array}$ & $\begin{array}{l}\text { Dialyzer } \\
\text { surface area } \\
\left(\mathbf{m}^{2}\right)\end{array}$ & $\boldsymbol{K t / V}$ \\
\hline 1 & $24-28$ & 3.9 & 250 & 0.66 & Polysulfone & 2.6 & 1.56 \\
2 & $24-28$ & 4.2 & 220 & $0.42-0.63$ & Polysulfone & 1.5 & 1.53 \\
3 & $24-28$ & 4.0 & $190-220$ & 0.55 & Polysulfone & 2.1 & 1.16 \\
4 & $24-28$ & 4.0 & 200 & 0.33 & Polysulfone & 1.5 & 1.65 \\
5 & $24-28$ & 4.0 & 200 & 0.45 & Polysulfone & 1.8 & 1.42 \\
6 & $24-28$ & 4.0 & 230 & 0.5 & Polysulfone & 2.5 & 1.29 \\
7 & $24-28$ & 4.0 & 200 & $0.3-0.5$ & Polysulfone & 1.1 & 1.50 \\
8 & $24-28$ & 4.2 & 150 & 0.43 & Polysulfone & 1.5 & 1.04 \\
9 & $24-28$ & 4.0 & 200 & 0.58 & Polysulfone & 1.3 & 1.52 \\
Mean & - & $4.0(0.1)$ & $206.9(27.4)$ & $0.50(0.10)$ & - & $1.8(0.5)$ & 1.41 \\
$(\mathrm{SD})$ & & & & & & & $(0.20)$ \\
\hline
\end{tabular}

$H D$ hemodialysis, $K t / V$ normalized dialysis dose, $S D$ standard deviation

strong negative correlation between EP and $K t$ / $V(r=-0.75) \quad$ (C) was found, whereas the amount removed was positively correlated with
$K t / V \quad(r=0.55) \quad(\mathrm{E})$. No correlations between these parameters and serum albumin were found (B, D, F). As for R5, the correlations 

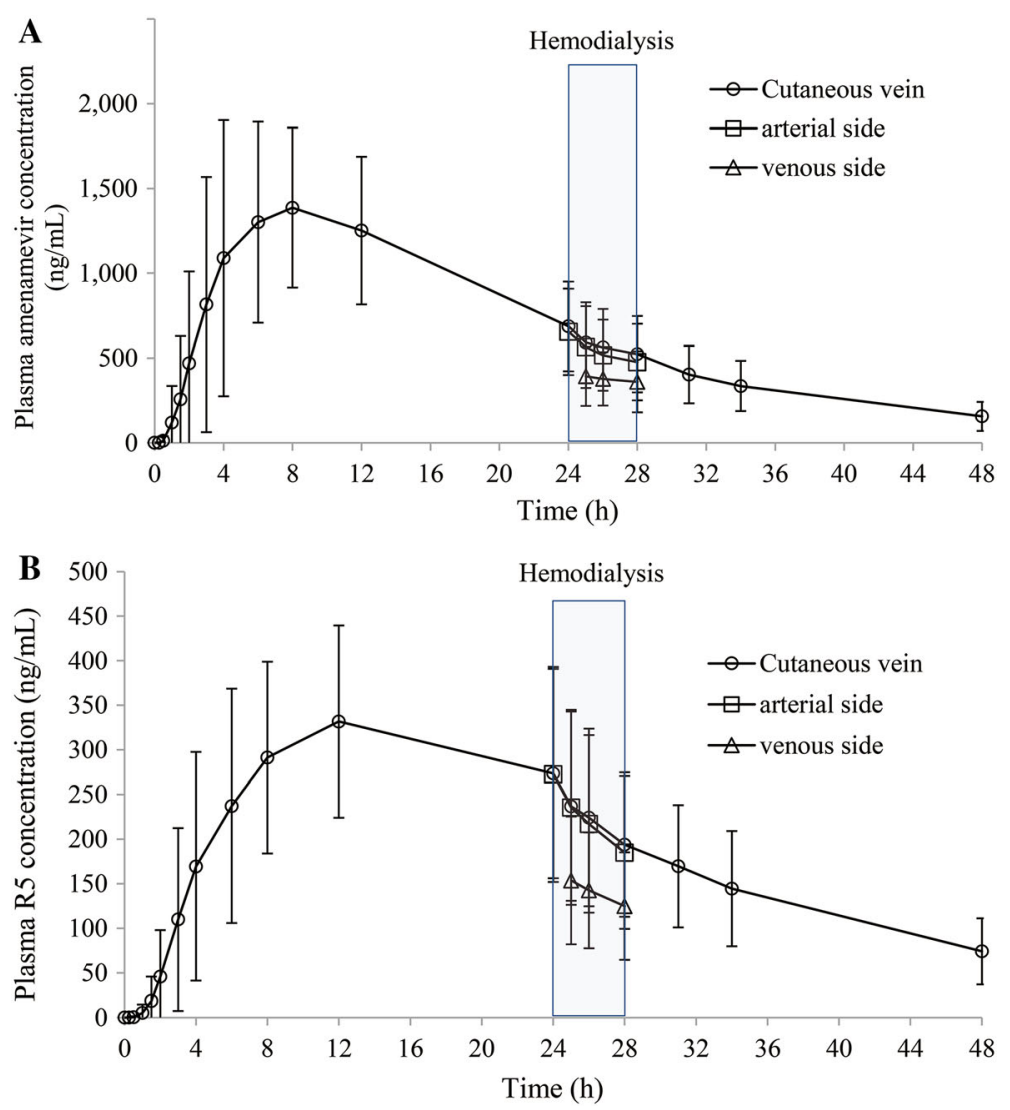

Fig. 2 Mean plasma concentration-time profiles after a single oral dose of amenamevir; a amenamevir, $\mathbf{b}$ its major metabolite R5

between dialyzability and either $K t / V$ or serum albumin are shown in Supplemental Fig. S2A-F. There was no correlation between $\mathrm{CL}_{\mathrm{HD}}$ and $\mathrm{Kt}$ / $V(\mathrm{~A})$, a strong negative correlation between EP and $K t / V(r=-0.74)(\mathrm{C})$, and a correlation between the amount removed and $K t /$ $V(r=0.69)$ (E). No correlations between dialyzability of R5 and serum albumin were found $(\mathrm{B}, \mathrm{D}, \mathrm{F})$.

\section{Safety}

There were no deaths or serious adverse events. No patients discontinued their participation in this study as a result of an adverse event. The only adverse event observed was abdominal distension in one patient. Any causal relationship with amenamevir was ruled out. No notable changes in clinical laboratory tests, vital signs, or weight were observed during the study.

\section{DISCUSSION}

In this study, we directly evaluated the pharmacokinetics and dialyzability of amenamevir and its major metabolite R5 in patients undergoing maintenance hemodialysis, and demonstrated that there were no clinically relevant safety concerns in the absence of dose adjustment and/or modification of the dosing schedule.

The pharmacokinetics and pharmacodynamics of nucleoside antivirus agents are affected significantly by kidney function. The AUC of penciclovir, a metabolite of famciclovir, in patients with severe renal impairment was approximately 8.7 times higher than in healthy participants with normal renal function, and modification of the dosing schedule and dose reduction are needed depending on the degree of renal function impairment [16]. On the other 
Table 3 Pharmacokinetic parameters of amenamevir and its major metabolite R5

\begin{tabular}{|c|c|c|c|c|c|c|}
\hline & \multicolumn{3}{|c|}{ Amenamevir } & \multicolumn{3}{|c|}{ R5 } \\
\hline & $n$ & Mean (SD) & Median (Min-Max) & $\bar{n}$ & Mean (SD) & Median (Min-Max) \\
\hline$C_{\max }(\mathrm{ng} / \mathrm{mL})$ & 9 & $1585(442)$ & $1658(888-2407)$ & 9 & $337.6(104.7)$ & $347.0(215.8-486.9)$ \\
\hline$t_{\max }(\mathrm{h})$ & 9 & $6.2(2.5)$ & $6(4-12)$ & 9 & $12.9(4.4)$ & $12(8-24)$ \\
\hline $\mathrm{AUC}_{24 \mathrm{~h}}(\mathrm{ng} \mathrm{h} / \mathrm{mL})$ & 9 & $23,890(8400)$ & $22,310(12,370-40,280)$ & 9 & $6054(2125)$ & $5830(3855-9765)$ \\
\hline $\mathrm{AUC}_{\text {inf }}(\mathrm{ng} \mathrm{h} / \mathrm{mL})$ & 7 & $38,120(17,180)$ & $34,100(16,210-65,970)$ & 0 & - & - \\
\hline$t_{1 / 2 \text { pre }}(\mathrm{h})$ & 7 & $13.9(2.6)$ & $14.7(9.9-17.0)$ & 0 & - & - \\
\hline$t_{1 / 2 \mathrm{HD}}(\mathrm{h})$ & 9 & $32.7(47.7)$ & $15.2(7.5-158.3)$ & 9 & $15.2(13.5)$ & $11.9(7.0-49.8)$ \\
\hline$t_{1 / 2 \text { post }}(\mathrm{h})$ & 9 & $12.0(2.5)$ & $12.4(8.4-15.7)$ & 9 & $14.0(2.8)$ & $15.0(9.3-17.2)$ \\
\hline $\mathrm{AUC}_{\mathrm{D}}(\mathrm{ng} \mathrm{h} / \mathrm{mL})$ & 9 & $2301(926)$ & $2041(808.7-3626)$ & 9 & $903.8(392.6)$ & $1025(370.9-1561)$ \\
\hline $\operatorname{AUC}_{\mathrm{A} 24-28 \mathrm{~h}}(\mathrm{ng} \mathrm{h} / \mathrm{mL})$ & 9 & $2145(905)$ & $2004(724.8-3555)$ & 9 & $882.2(401.6)$ & $1021(351.2-1571)$ \\
\hline $\mathrm{AUC}_{\mathrm{V} 24-28 \mathrm{~h}}(\mathrm{ng} \mathrm{h} / \mathrm{mL})$ & 9 & $1509(672)$ & $1252(435.7-2451)$ & 9 & $565.7(262.4)$ & $672.9(193.5-964.2)$ \\
\hline $\mathrm{Ae}_{24 \mathrm{~h}}(\mathrm{mg})$ & 5 & $1.6(0.8)$ & $1.8(0.7-2.7)$ & 5 & $0.9(0.9)$ & $0.8(0.2-2.4)$ \\
\hline $\mathrm{Ae}_{24-48 \mathrm{~h}}(\mathrm{mg})$ & 5 & $0.4(0.3)$ & $0.2(0.1-0.8)$ & 5 & $0.4(0.3)$ & $0.5(0.1-0.8)$ \\
\hline $\mathrm{fb}_{4 \mathrm{~h}}(\%)$ & 8 & $64.2(3.3)$ & $63.6(61.3-72.0)$ & - & - & - \\
\hline $\mathrm{fb}_{24 \mathrm{~h}}(\%)$ & 9 & $62.7(4.0)$ & $62.2(58.5-72.5)$ & - & - & - \\
\hline
\end{tabular}

$A e_{24 h}$ urine pharmacokinetic parameters excreted into the urine from time zero to $24 \mathrm{~h}, A e_{24-48 \mathrm{~h}}$ Ae from time 24 to $48 \mathrm{~h}$ (Ae24-48 h), $A U C_{24 h}$ area under the plasma concentration-time curve from time zero to $24 \mathrm{~h}, A U C_{A 24-28 \mathrm{~h}}$ AUC during hemodialysis calculated on the basis of the concentration at the inlet of the dialyzer, $A U C_{D}$ AUC during hemodialysis, $A U C_{\text {inf }}$ AUC from time zero to infinity, $A U C_{V 24-28 h}$ AUC during hemodialysis calculated on the basis of the concentration of the outlet of the dialyzer, $C_{\max }$ maximum plasma concentration, $f b_{4 b}$ plasma protein binding $\%$ at $4 \mathrm{~h}$ post-dose, $f b_{24 b} \mathrm{fb}$ at $24 \mathrm{~h}$ post-dose, Max maximum, Min minimum, $S D$ standard deviation, $t_{1 / 2 H D}$ terminal elimination half-life $\left(t_{1 / 2}\right)$ during hemodialysis, $t_{1 / 2 \text { pre }} t_{1 / 2}$ observed within $24 \mathrm{~h}, t_{1 / 2 p o s t} t_{1 / 2}$ after hemodialysis, $t_{\max }$ time to reach maximum plasma concentration during $24 \mathrm{~h}$ post-dose

hand, amenamevir is mainly metabolized in the liver and urinary excretion of the unchanged amenamevir was approximately $10 \%$ in healthy participants with normal kidney function [5]. The $C_{\max }$ of amenamevir in hemodialysis patients $(1585 \mathrm{ng} / \mathrm{mL})$ was similar to that in healthy adults $(1612 \mathrm{ng} / \mathrm{mL})$. The $\mathrm{AUC}_{\text {inf }}$ of amenamevir in hemodialysis patients $(38,120 \mathrm{ng} \mathrm{h} / \mathrm{mL})$ was slightly higher than that reported in non-dialysis patients with severe renal impairment $(30,621 \mathrm{ng} \mathrm{h} / \mathrm{mL})$, and approximately double that of healthy adults $(16,886 \mathrm{ng} \mathrm{h} / \mathrm{mL})$ [6]. The $t_{1 / 2}$ of amenamevir was slightly longer in patients with mild $(8.4 \mathrm{~h})$, moderate $(9.5 \mathrm{~h})$ and severe $(9.8 \mathrm{~h})$ renal impairment compared with that in participants with normal renal function $(8.1 \mathrm{~h})$ [6]. The median $t_{1 / 2 \text { pre, }} t_{1 / 2 \mathrm{HD}}$, and $t_{1 / 2 \text { post }}$ of amenamevir in this study were $14.7,15.2$, and $12.4 \mathrm{~h}$, respectively, suggesting that $t_{1 / 2}$ is prolonged in proportion to the extent renal function impairment. Hemodialysis had a greater effect on the disposition of R5 than amenamevir as was also shown in non-dialysis patients with CKD [6]. The AUC and $C_{\max }$ of $\mathrm{R} 5$ in hemodialysis patients were respectively almost triple and double those reported in participants with normal renal function [6]. They were higher than in non-dialysis patients with severe renal impairment, suggesting that renal 


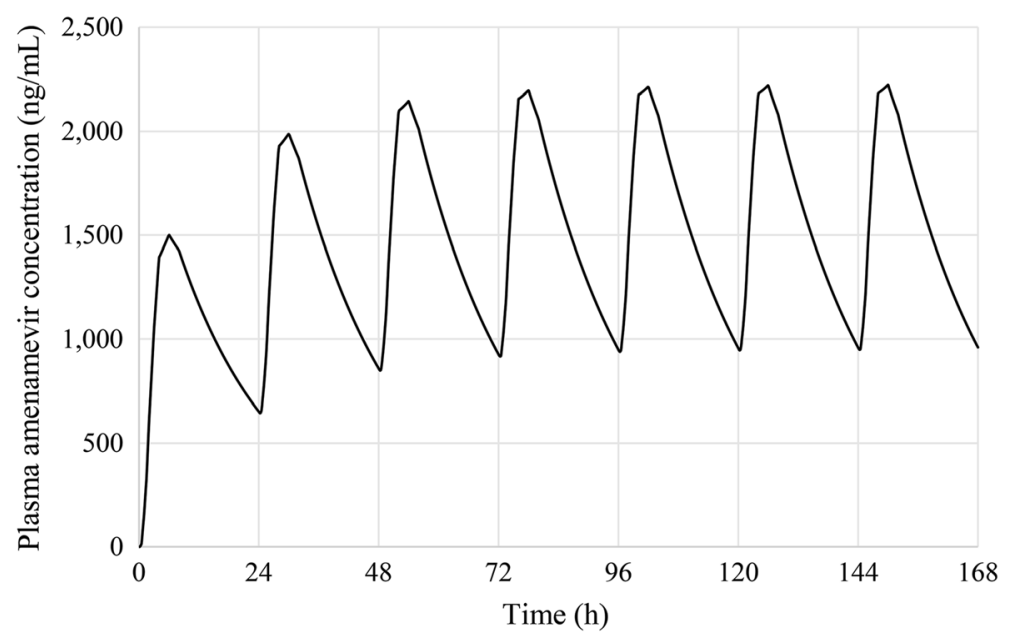

Fig. 3 Simulation of the plasma concentration-time curve of multiple oral doses of amenamevir calculated on the basis of seven patients under the assumption that no amenamevir was removed by hemodialysis

Table 4 Hemodialysis parameters of amenamevir and its major metabolite R5 $(n=9)$

\begin{tabular}{|c|c|c|c|c|c|c|}
\hline & \multicolumn{3}{|c|}{ Amenamevir } & \multicolumn{3}{|l|}{ R5 } \\
\hline & $\begin{array}{l}\text { Mean } \\
(S D)\end{array}$ & Median & $\operatorname{Min-Max}$ & $\begin{array}{l}\text { Mean } \\
\text { (SD) }\end{array}$ & Median & $\overline{M i n-M a x}$ \\
\hline Hemodialysis clearance $(\mathrm{mL} / \mathrm{min})$ & $\begin{array}{l}37.8 \\
(5.3)\end{array}$ & 37.5 & $30.9-48.0$ & $\begin{array}{l}46.5 \\
(4.4)\end{array}$ & 45.0 & $41.4-56.2$ \\
\hline $\begin{array}{l}\text { Hemodialysis clearance normalized with dialyzer } \\
\text { surface area }\left(\mathrm{mL} / \mathrm{min} / \mathrm{m}^{2}\right)\end{array}$ & $\begin{array}{l}22.7 \\
(5.4)\end{array}$ & 22.8 & $15.1-32.3$ & $\begin{array}{l}28.2 \\
(7.4)\end{array}$ & 27.6 & $18.2-40.8$ \\
\hline Elimination fraction percentage (\%) & $\begin{array}{l}28.1 \\
(5.1)\end{array}$ & 27.4 & $22.2-36.6$ & $\begin{array}{l}34.5 \\
(4.7)\end{array}$ & 32.5 & $29.6-42.9$ \\
\hline Removed amount $(\mu \mathrm{g})$ & $\begin{array}{l}132.0 \\
(54.2)\end{array}$ & 131.6 & $43.4-236.8$ & $\begin{array}{l}65.1 \\
(28.7)\end{array}$ & 69.6 & $23.7-121.4$ \\
\hline
\end{tabular}

Max maximum, Min minimum, SD standard deviation

elimination might be the predominant pathway for metabolite R5. However, R5 is not an active metabolite [6]; therefore, the increased exposure of R5 in hemodialysis patients might be less clinically meaningful. The recommended oral dosage of amenamevir is $400 \mathrm{mg}$ once a day after a meal for 7 days. The simulated plasma concentration-time curve of 7-day administration of amenamevir in hemodialysis patients without taking into account drug removal by dialysis indicated that the $C_{\max }$ increases slightly during the first 3 days post-dose, then remains steady during the following 4 days. This suggests that the risk of overdose resulting from multiple doses would be very low. As described later, the amount of drug removed by dialysis was limited. Amenamevir was safe and well tolerated when administered as single doses from 5 to $2400 \mathrm{mg}$ under fasting conditions, and a dose of 300 or $600 \mathrm{mg}$ once a day for 7 days under fed conditions were administered [2]. Even though systemic amenamevir exposure doubled in hemodialysis patients, the exposure in these patients was within the range 
reported in previous pharmacokinetic studies which demonstrated the drug's safety and tolerability [2]. Thus, repeated dosing of $400 \mathrm{mg}$ amenamevir once a day for 7 days in hemodialysis patients is unlikely to cause significant safety-related problems. Therefore, dose reduction is not required in these patients.

The difference between $\mathrm{AUC}_{\mathrm{A} 24-28 \mathrm{~h}}$ and AUC $_{\mathrm{V} 24-28 \mathrm{~h}}$ suggests that about $30 \%$ of amenamevir was removed by hemodialysis. This low level of removal was also confirmed by the result that the median $t_{1 / 2}$ during dialysis was not shortened compared to that before and after dialysis. These findings suggest that removal of amenamevir by dialysis is limited, and additional administration after dialysis and/or change in the dosing schedule is not needed. On the other hand, additional dosing is required in the case of acyclovir after hemodialysis, since about $70 \%$ of acyclovir is removed by 4 -h hemodialysis [17]. We investigated whether there was a correlation between the dialysis parameters including $\mathrm{CL}_{\mathrm{HD}}, \mathrm{EP}$, and the removed amount, and $K t / V$ and serum albumin to elucidate the factors affecting the dialyzability of amenamevir, but only a slight correlation was found between the removed amount and $K t / V$.

This study has several limitations. First, the data from patients in poor general condition undergoing hemodialysis were not available because such patients were excluded from this study because of the unacceptable burden imposed by frequent blood samplings. Second, the study population was relatively small as is the case in most other phase 1 studies, and this study did not attempt to make a direct comparison of the pharmacokinetics of amenamevir between hemodialysis patients and healthy participants or participants with renal impairment. Third, hemodialysis patients with herpes zoster were not enrolled in this study. Last, the simulation of pharmacokinetics of multiple doses of amenamevir was calculated solely on the basis of the pharmacokinetics of a single oral dose. The effect of enzyme induction on the pharmacokinetics was not considered. Therefore, ongoing collection of information on hemodialysis patients in the real world is needed to confirm the efficacy and safety of amenamevir in hemodialysis patients.

\section{CONCLUSION}

We directly evaluated the pharmacokinetics of amenamevir following the administration of a single oral dose of $400 \mathrm{mg}$ given after breakfast to patients with ESKD undergoing hemodialysis. The AUC of amenamevir was approximately doubled compared to healthy adults. This increase was much smaller than those reported in hemodialysis patients treated with nucleoside antiviral agents [8-10]. The amount of amenamevir removed by hemodialysis was minimal. There were no clinically relevant safety concerns after the usual dosage (400 mg/day) of amenamevir was administered to hemodialysis patients in the absence of dose adjustment and/or modification of the dosing schedule. Therefore, amenamevir can be administered once daily either before or after dialysis.

\section{ACKNOWLEDGEMENTS}

We thank all participating patients and their families, the study investigators (Supplementary Material), study nurses, study monitors, data manager, Rumiko Kato and all other members of this study team.

Funding. This study and the journal's Rapid Service and Open Access fees were funded by Maruho Co., Ltd.

Medical Writing Assistance. We would like to thank Tetsuji Asao, PhD, SunFlare Co., Ltd., Tokyo, Japan, who wrote the first draft of this manuscript. This medical writing assistance was funded by Maruho Co., Ltd.

Authorship. All named authors meet the International Committee of Medical Journal Editors (ICMJE) criteria for authorship for this article, take responsibility for the integrity of the work as a whole, and have given their approval for this version to be published. 
List of Investigators. A full list of the investigators for this study can be found in the Supplementary Material.

Disclosures. Shuichi Tsuruoka received honoraria and consultant fee from Maruho Co., Ltd. The current affiliation of Shuichi Tsuruoka is Tajirigaoka Hospital (Hitachi, Ibaraki, Japan). Takamasa Endo, Mizuna Seo, and Naoto Hashimoto are employees of Maruho Co., Ltd.

Compliance with Ethics Guidelines. The study protocol and informed consent form were approved by the institutional review board at each participating study site (Supplementary Information). All patients gave written informed consent before initiation of any study specific procedures. The study was designed and conducted by the sponsor (Maruho Co., Ltd.) in collaboration with the principal investigators. The sponsor monitored study conduct, collected the data, and performed the statistical analyses. The study was conducted in accordance with the ethical principles originating in or derived from the Declaration of Helsinki, International Conference on Harmonisation Good Clinical Practice Guidelines, and locally applicable laws and regulations. This study is registered with the Japan Pharmaceutical Information Center; JapicCTI-184242.

Data Availability. The datasets generated during and/or analyzed during the current study are available from the corresponding author on reasonable request.

Open Access. This article is licensed under a Creative Commons Attribution-NonCommercial 4.0 International License, which permits any non-commercial use, sharing, adaptation, distribution and reproduction in any medium or format, as long as you give appropriate credit to the original author(s) and the source, provide a link to the Creative Commons licence, and indicate if changes were made. The images or other third party material in this article are included in the article's Creative Commons licence, unless indicated otherwise in a credit line to the material. If material is not included in the article's Creative Commons licence and your intended use is not permitted by statutory regulation or exceeds the permitted use, you will need to obtain permission directly from the copyright holder. To view a copy of this licence, visit http://creativecommons.org/licenses/by$\mathrm{nc} / 4.0 /$.

\section{REFERENCES}

1. Chono K, Katsumata K, Kontani T, et al. ASP2151, a novel helicase-primase inhibitor, possesses antiviral activity against varicella-zoster virus and herpes simplex virus types 1 and 2 . J Antimicrob Chemother. 2010;65:1733-41.

2. Kusawake T, Keirns JJ, Kowalski D, et al. Pharmacokinetics and safety of amenamevir in healthy subjects: analysis of four randomized phase 1 studies. Adv Ther. 2017;34:2625-37.

3. Ohtsu Y, Susaki Y, Noguchi K. Absorption, distribution, metabolism, and excretion of the novel helicase-primase inhibitor, amenamevir (ASP2151), in rodents. Eur J Drug Metab Pharmacokinet. 2018;43:693-706.

4. Kato K, den Adel M, Groenendaal-van de Meent D, Ohtsu Y, Takada A, Katashima M. An open-label, single-dose, human mass balance study of amenamevir in healthy male adults. Clin Pharmacol Drug Dev. 2019;8:595-602.

5. Ohtsu Y, van Trigt R, Takama K, et al. Quantification of ASP2151 in human plasma and urine: a pitfall associated with supersaturation of analyte in urine. Chromatographia. 2017;80:217-27.

6. Kusawake T, Kowalski D, Takada A, et al. The influence of hepatic and renal impairment on the pharmacokinetics of a treatment for herpes zoster, amenamevir (ASP2151): phase 1, open-label, singledose, parallel-group studies. Adv Ther. 2017;34: 2612-24.

7. Package insert of Amenalief ${ }^{\circledR}$. 2018. http://www. info.pmda.go.jp/go/pdf/730155_6250046F1028_1_ 03 (in Japanese). Accessed Feb 19, 2020.

8. Package insert of ZOVIRAX ${ }^{\circledR}$. 2005. https://www. accessdata.fda.gov/drugsatfda_docs/label/2005/ 018828s030,020089s019,019909s020lbl.pdf. Accessed Feb 19, 2020.

9. Package insert of VALTREX $^{\circledR} \quad$ (valacyclovir hydrochloride) Caplets. 2008. https://www.accessdata.fda.gov\%2Fdrugsatfda_docs $\% 2$ Flabel\% 2F2008\%2F020487s014lbl.pdf\&usg= 
AOvVaw3SbPEplJCUHPX3-H7cEORr. Accessed Feb 19,2020 .

10. Package insert of FAMVIR (famciclovir) tablets. 2018. https://www.accessdata.fda.gov/drugsatfda docs/label/2018/020363s050lbl.pdf. Accessed Feb $19,2020$.

11. Velenosi TJ, Urquhart B. Pharmacokinetic considerations in chronic kidney disease and patients requiring dialysis. Expert Opin Drug Metab Toxicol. 2014;10:1131-43.

12. Lee CS, Marbury TC. Drug therapy in patients undergoing haemodialysis. Clinical pharmacokinetic considerations. Clin Pharmacokinet. 1984;9: 42-66.

13. Gabutti L, Taminelli-Beltraminelli L, Marone C. Clearance of ceftriaxone during haemodialysis using cuprophane, haemophane and polysulfone dialysers. Eur J Clin Pharmacol. 1997;53:123-6.
14. Hasegawa G, Tsuruoka S, Ushijima K, et al. Dialyzability of faropenem in infected patients on chronic hemodialysis. Ther Apher Dial. 2017;21:52-6.

15. Daugirdas JT. Second generation logarithmic estimates of single-pool variable volume $\mathrm{Kt} / \mathrm{V}$ : an analysis of error. J Am Soc Nephrol. 1993;4: 1205-13.

16. Boike SC, Pue MA, Freed MI, et al. Pharmacokinetics of famciclovir in subjects with varying degrees of renal impairment. Clin Pharmacol Ther. 1944;55: 418-26.

17. Hara K, Suyama K, Itoh H, Nagashima S. Influence of ALDH2 genetic polymorphisms on aciclovir pharmacokinetics following oral administration of valaciclovir in Japanese end-stage renal disease patients. Drug Metab Pharmacokinet. 2008;23: 306-12. 